\title{
A Tidal Trigger of Starburst or Seyfert Activity
}

\author{
Tapan K. Chatterjee \\ Facultad de Ciencias, Fisico-Matematicas, Universidad A. Puebla, A. \\ P. 1316, Puebla, Mexico
}

\section{Introduction and Theory}

Some of the classical seyferts are observed to be prototypical ovals, e.g. NGC 1068 and 4151 (e.g., Bosma 1981; Kormendy 1982; Scoville et al. 1988). Such a non-axisymmetric potential corresponding to an oval disk can produce inward flow of gas and induce mild activity. To test the efficiency of this process, we study a collision between a face-on spiral with a high gaseous content and an equally massive compact elliptical, under marginally bound conditions, as such encounters are most frequent.

We model the spiral galaxy by an exponential model disk (of radius $R$ ) with a (static) thickness and scale length $\alpha=4 / R$ and a spherical polytropic bulge ( $n=0,3,4$, equally weighted combination) containing $1 / 3$ of the mass (cf. Chatterjee 1990); about $20 \%$ of the mass of the disk contains gas particles. The elliptical is modeled identically as the bulge. The gravitational potential is softened with softening constants of $\epsilon=r_{o} / 5, r_{o} / 3$, and $0.8 r_{o}$, for the bulge of the spiral as well as the elliptical, stellar and gaseous components of the disk, respectively. Here $r_{o}$ is the radius containing $75 \%$ of the total mass of the galaxy in question, while the mutual gravitational interaction is softened with a softening constant of $r_{o} / 4$.

The Lagrangian Equations (in polar coordinates $r$ and $\theta$ ) are used to determine the relative orbit of the perturber (Galaxy p) with respect to the test system (Galaxy t): $d t=\left[2 \mu^{-1}\left(E-W(r)-\frac{L^{2}}{2 \mu r^{2}}\right)\right]^{-0.5}$, and $d \theta=\frac{L}{\mu r^{2}} d t$, where $\mu, L$ and $E$ are the reduced mass, angular momentum and the orbital energy, respectively, and $W(r)$ is the instantaneous potential energy of interaction between the galaxies. At small intervals of $r$ and $\theta$, the tidal effects and the corresponding changes in velocities of the stars are determined, such that the instantaneous internal energy changes of the two galaxies $\Delta U_{p}(t)$ and $\Delta U_{t}(t)$ can be determined; the instantaneous relative velocity follows from $V(t)=$ $\left[2 \mu^{-1}\left(E_{i}-W(t)-\Delta U_{p}(t)-\Delta U_{t}(t)\right)\right]^{-0.5}, E_{i}$ being the initial value of $E$. The galaxies are interchanged at each interval to determine the relevant quantities for each one (cf. Chatterjee 1992). All the particles are initially given circularly symmetric motion. The three-body approach is adopted to study the motion of the softened stellar and gaseous particles. At the closest approach, the disk of the spiral galaxy attains an oval shape pointed towards the perturber (with an equatorial axial ratio $\approx 0.7$ ). We calculate the changed mass distribution of the oval disk and then its potential as well as the mutual potential energy of the galaxy pair (at this pericentric position) from first principles, thereafter contin- 


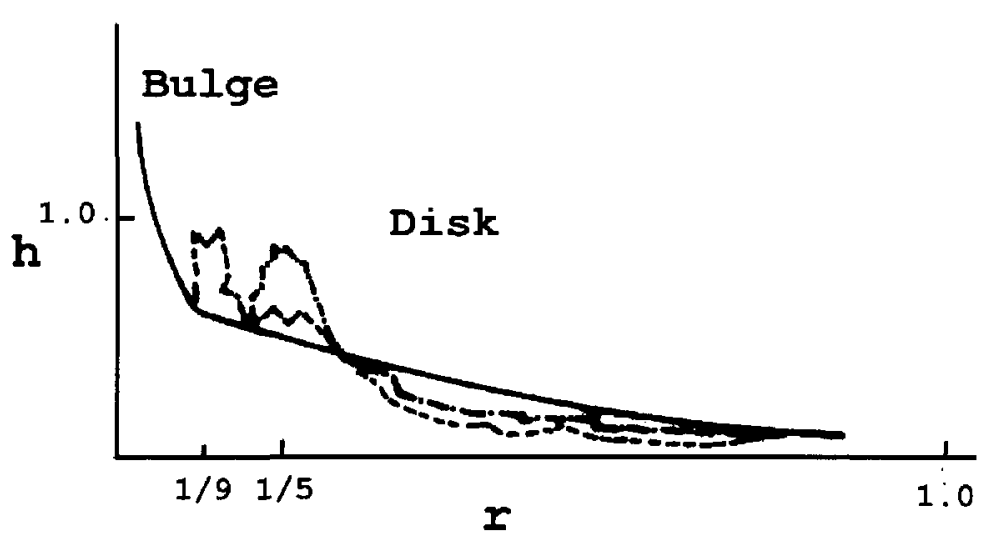

Figure 1. Evolution of the density with radius for the model described in the text. The solid, dash-dot-dash, and dashed curves are for $t=-1.0,0.1$, and 10 , respectively.

uing the study of the motion of the particles. (We do not consider the rotation of the galaxies.)

\section{Results and Conclusions}

The external tidal forcing causes a torque, which has its maximum effect in the vicinity of the pericentric approach; the external torquing causes the (lighter) gas (particles) in the outer regions to gain energy and angular momentum and their orbits expand, while the inner particles are hardly affected. However, the (heavier) stellar constituent of the disk is distorted into an oval (with an equatorial axial ratio $\approx 0.7$ ), with its sharp end pointing towards the elliptical perturber; this end is also substantially enhanced in density at the cost of the broad end of the oval, which gets spread. The influence of the density enhancement at the sharp end is accentuated due to the presence of the perturber in that region. The disk behaves in a manner very similar to one having an induced bar; in fact, its modified potential is very similar to that of a disk with a prototypical $\cos 2 \theta$ bar. Subsequently the gas feels the modified force due to the oval distortion and the gas in the outer regions moves inwards in elongated orbits, while the gas in the inner regions is hardly affected and maintains almost circular motion. At this stage the outer gas falls on the inner gas, forming a ring-like density enhancement and there are collisions and exchange of angular momentum; we assume the collisions to be $50 \%$ inelastic. The orbits are followed, with the modified elements, after each collision. The ring now breaks up as the gas slowly spirals inwards due to loss of energy on account of the inelastic collisions.

We use a dimensionless system of units in which the total mass and radius of the galaxies are taken as unity and $\mathrm{G}=0.0450$ (such that a translation to physical units for the collision in which each galaxy has a mass of $10^{11}$ solar 
masses and radius $10 \mathrm{kpc}$, gives the unit of time as $10^{7}$ years). We show the results graphically in Figure 1, where the numerical density, $n$, is shown as a function of the radial distance, $r$, for the initial time $t=-1.0$, shortly after the pericentric approach $(t=0.1)$ and after the formation of the accretion disk $(t=$ 2 ). Both $n$ and $r$ are normalized with respect to their maximum values in the disk component of the spiral.

However, the gas does not fall to the center, but acquires a quasi-stable configuration in near circular orbits in a central region of radial extent $\approx 0.1 R$, where it forms an accretion disk. However, in the presence of a supermassive black hole, instability can be induced to this accretion disk, causing an infall of gas to the nucleus. After the perturber has receded to a long distance the disk loses its oval distortion and there is a secondary mass distribution change, but of markedly lesser magnitude.

Acknowledgments. It is a matter of great pleasure to thank the organizers of the Colloquium for their excellent hospitality and organization.

\section{References}

Bosma, A. 1981, AJ, 80, 1825

Chatterjee, T. K. 1990, Ap\&SS, 163, 127

Chatterjee, T. K. 1992, Ap\&SS, 196, 283

Kormendy, J. 1982, in Morphology and Dynamics of Galaxies, L. Martinet \& M. Mayor, Sauverny: Geneva Obs., 113

Scoville, N., Matthews, K., Cario, D. P., \& Sanders, D.P. 1988, ApJ, 327, L61 